\title{
LA FORMACIÓN EN ECONOMÍA SOCIAL. ANÁLISIS DE LA OFERTA UNIVERSITARIA DE POSGRADO EN ESPAÑA
}

\author{
POR \\ David FLORES RUIZ, \\ Carmen GUZMÁN ALFONSO y \\ María de la O BARROSO GONZÁLEZ ${ }^{1}$
}

\section{RESUMEN}

En el presente artículo se analiza la importancia que tiene la formación para el fomento de la economía social, prestando especial atención a la caracterización de la oferta formativa de este tipo de estudios y diferenciándola respecto a la oferta formativa convencional en el área de Administración y Dirección de Empresas, emprendimiento e innovación. Previo a ello, se reflexiona, en base a un breve análisis bibliográfico, sobre las relaciones que se establecen entre educación y economía para, posteriormente, centrarnos en la caracterización de este tipo de formación, prestando especial atención a la que se centra en la Economía Social. Para ello, en primer lugar, se ha elaborado una base de datos en la que se incluyen todas las titulaciones de posgrado relacionadas con los estudios de Economía. Asimismo, se ha diseñado un cuestionario estructurado con el objetivo de caracterizar este tipo de formación. Se concluye la escasa importancia que tiene en el sistema universitario de posgrado español la formación en Economía Social, detectándose algunas diferencias significativas en la caracterización del proceso formativo entre las titulaciones de Economía Social y las convencionales de Administración y Dirección de Empresas, emprendimiento e innovación empresarial, en aspectos tales, como: los valores, las competencias, las habilidades, la forma de entender y transmitir el funcionamiento de la economía, etc. A partir de estas conclusiones se dan una serie de recomendaciones que pueden contribuir a fomentar y dinamizar, mediante la formación en posgrados, la Economía Social.

1 Departamento de Economía de la Universidad de Huelva. Direcciones de correo electrónico: David.flores@dege.uhu.es, Carmen.guzman@dege.uhu.es, barroso@uhu.es.

REVESCO N 121 - Segundo Cuatrimestre 2016 - ISSN: 1885-8031 - www.ucm.es/info/revesco

http://dx.doi.org/10.5209/rev_REVE.2016.v121.49703

Fecha de recepción: 20/02/2015

Fecha de aceptación: 28/07/2015 
Palabras clave: Estudios Económicos, Economía Social, Educación Superior, Estudios de Posgrados, Ciencias Sociales.

Claves ECONLIT: A20, A29, I23, I25.

\title{
THE TRAINING IN SOCIAL AND SOLIDARITY ECONOMY. ANALYSIS OF THE UNIVERSITY OFFER IN SPAIN
}

\begin{abstract}
This research analyses the importance of the training for the promotion of the social economy in two ways: 1) focusing on the characteristics of the educational offer of Social Economy and 2) analyzing its differences regarding to those studies from the Management, entrepreneurship, and innovations areas. To this aim, it is carried out as a first step a literature review of the contributions that study the relationships that can exist between the education and Economy. Then, being based on this previous analysis, we already center our research on the Social Economy sector and its relations with the education system. To get this objective, it has been developed a database that includes all the postgraduate titles related to the Economics area. Likewise, a questionnaire has been designed with the aim of characterize the training in social Economy. As a conclusion, it is obtained that the training in social economy in postgraduate studies in the Spanish Universities is very poor. On the other hand, there are significant differences between Social Economy degrees and Business Management, entrepreneurship and innovation degrees with regard to different aspects such as: values to transmit, competencies, skills, the way of understanding the Economy, etc. Based on these conclusions, different recommendations are proposed in order to promote and boost this other way of doing Economy through the training and education in postgraduate studies.
\end{abstract}

Keywords: Economic Studies, Social Economy, Higher Education, postgraduate studies, Social Sciences.

\section{INTRODUCCIÓN}

Podría decirse que la educación, es decir, la institución educativa, tiene un marcado carácter socio-político, ya que su configuración va a determinar, influir y modelizar a la sociedad en la que se inserta. No es, por tanto, una institución neutra y ajena a la propia sociedad. No es una institución natural que nadie puede cuestionar, por lo que su 
configuración y funcionamiento siempre estará abierta a procesos de reflexión y debates (Martínez Rodríguez, 2013).

Por su parte, la Economía, en cuanto a ciencia social, también está sujeta a valores y comportamientos, ya que no debe olvidarse que proviene de la rama de la Filosofía Moral, siendo, por tanto, consustancial a la Ética y los valores, lo cual hace que su construcción también esté abierta a procesos de reflexión y debates (Miedes Ugarte y Flores Ruiz, 2013).

En este sentido, el presente artículo, a partir de una breve reflexión teórica sobre la formación en Economía, y en Economía Social en particular, trata de poner de manifiesto el tipo de formación que reciben en España los profesionales en Economía y Administración y Dirección de Empresas, a partir del análisis de los estudios de posgrados ofertados por el sistema universitario español. De tal forma, que el objetivo central del presente artículo es el de poner de manifiesto las diferencias que puedan existir entre la formación convencional en el área de las Ciencias Económicas y Empresariales respecto a la formación de la Economía Social.

No en vano, como hipótesis de trabajo, se parte de que el tipo de formación de posgrado en esta área de las Ciencias Económicas y Empresariales descansa en el paradigma ortodoxo, pudiéndose identificar en estos programas formativos diferencias significativas respecto a los que se centran en la denominada Economía Social.

Para validar esta hipótesis se analiza la oferta formativa española de posgrados de esta área con el fin de comparar aquella oferta que sigue una orientación más ortodoxa respecto a la que se ocupa principalmente de la Economía Social, con objeto de destacar posibles diferencias en lo que a aspectos cuantitativos y cualitativos respecta. En última instancia, a modo de conclusión, se proponen determinadas actuaciones genéricas que podrían contribuir a dinamizar este tipo de economía.

\section{FUNTAMENTACIÓN TEÓRICA}

\subsection{Formación y Economía. Una caracterización de la formación en Economía}

La escuela, como espacio para la socialización de las clases populares, no es una institución que haya existido siempre, sino que se trata de una institución relativamente reciente que cuenta aproximadamente con un siglo de existencia. Así, para Valera y Álvarez- 
Uría (1991: 14), la escuela pública, gratuita y obligatoria ha sido instituida por Romanotes al principio del siglo XX, convirtiendo a los maestros en funcionarios del Estado.

La naturaleza de la escuela ha estado estrechamente ligada a la idea de control social, identificándose la educación con la socialización y, por tanto, como instrumento de control social. Así, Martínez Rodríguez (2013:45) afirma que las ideologías conservadoras utilizan los modelos neoliberales para configurar un sistema educativo que condicione los modos de pensar, con objeto de legitimar las estructuras de poder dominante, articulando un modelo de escuela para plasmar en la sociedad el "único" prototipo posible, según sus partidarios más ortodoxos, de ciudadanos, que concibe al sujeto como ser individualista, competitivo, materialista y consumista.

Pero la educación y la formación no sólo influyen en la economía mediante la socialización del ciudadano, también influye a la hora de formar a los propios economistas, en la medida en que sus pensamientos, ideologías y teorías son las que posteriormente se ponen en práctica a la hora de diseñar o influir en el diseño de las diferentes políticas e instituciones que modelan el funcionamiento de la economía; entre ellas, la del sistema educativo y los propios planes de estudios de las titulaciones que se integran en las Ciencias Económicas y Empresariales.

Como recoge Delgado Munevar (2012), el economista debe preocuparse por los problemas reales que le rodea, ya que al asumir esta actitud podrá conectarse con su propia realidad para identificar y diagnosticar problemas, y buscar soluciones a los mismos. De tal forma que la Economía se le suele denominar la ciencia de la praxis, en la medida en que su principal objetivo es generar un tipo de conocimiento que se oriente a la acción eficaz para resolver problemas sociales reales (Cuadrado Roura, 1995).

Sin embargo, a comienzos del presente siglo, un grupo de estudiantes de Economía de universidades francesas publicaron una carta abierta en la cual señalaban su descontento con la enseñanza recibida. Esta carta también fue acogida por universidades inglesas, alemanas, españolas e italianas. Sus críticas se centraban en tres puntos: a) el divorcio entre el contenido de la enseñanza y el "mundo real"; b) un uso incontrolado de las matemáticas; c) una falta de pluralismo en las explicaciones dadas (Misas Arango, 2004).

Aún así, a pesar de estos documentos, en los que queda recogido el divorcio creciente entre el contenido de la enseñanza de la Economía y "el mundo real", no se ha logrado 
generar dinámicas suficientemente fuertes para modificar la situación, ya que las presiones para mantener el status quo son demasiado fuertes (Leontieff, 1982). Esta falta de giro en los estudios sobre Economía queda plasmada en el hecho de que los únicos campos que se exigen en más de la mitad de las principales universidades de Estados Unidos y Europa en la titulación de Economía son: "Introducción a la Economía", "Microeconomía", "Macroeconomía”, "Matemáticas”, "Estadística” y "Econometría”, dominando, por tanto, los instrumentos cuantitativos, sobre las teorías (Monteiro y Ferreira, 2006).

Por su parte, para el caso español, cuando se analizan los planes de estudios de las titulaciones de Economía y Dirección y Administración de Empresas, recogidos en el Libro Blanco del título de Grado en Economía y Empresa, según la ANECA (2005), se observa que los conocimientos instrumentales que deben tener los graduados en Empresa y Economía van referidos también al manejo de técnicas cuantitativas, tales como: elementos de álgebra lineal, cálculo diferencial e integral, optimización matemática, matemática de las operaciones financieras, estadística descriptiva, probabilidad, inferencia estadística, modelos de regresión simple y de variables explicativas; no contemplándose instrumentos de corte cualitativo, tales como: análisis Delphi, dinámicas de grupos, técnicas de participación y liderazgo, etc.

Para confirmar esta supremacía, se puede observar cómo en los contenidos formativos mínimos se recoge un bloque de Métodos Cuantitativos para la Empresa (Empresa) y otro de Métodos Cuantitativos para la Economía (Economía), así como otro bloque, para ambos grados, de Análisis Económico (microeconomía y macroeconomía). Dentro de estos bloques no encontramos ninguno relacionado con las Ciencias Políticas, la Sociología, la Ética, el Desarrollo Económico, etc ${ }^{2}$.

Respecto a las competencias y habilidades destacan: entender las instituciones económicas como resultado y aplicación de representaciones teóricas a cerca de cómo funciona la economía (ANECA, 2005). Por lo tanto, tal y como se ha apuntado anteriormente, puede decirse que las instituciones económicas se construyen y diseñan según la teoría económica dominante. Sin embargo, debemos preguntarnos: ¿cuál es la teoría económica que se enseña? En este sentido, el tipo de teoría económica queda reflejada cuando analizamos los objetivos de formación y aprendizaje de conocimientos teóricos del grado de Economía. De forma que, a partir del análisis de los mismos, puede afirmarse cómo la teoría económica que

\footnotetext{
${ }^{2}$ Estos bloques temáticos, dentro de los cuales se contemplan los contenidos mínimos a desarrollar, son: Análisis Económico, Economía del Sector Público, Economía Española, Internacional y Sectorial, Entorno Empresarial, Métodos Cuantitativos para la Economía e Historia Económica y Marco Jurídico.
} 
se enseña es la teoría neoclásica basada en la maximización de la utilidad a través de los $\operatorname{precios}^{3}$.

Por su parte, entre los posgrados oficiales que propone el Libro Blanco y que están directamente relacionados con estos contenidos teóricos, se encuentran los siguientes: Actuariales, Contabilidad y Auditoría, Dirección y Administración de Empresa, Finanzas, Marketing, Análisis Económico, Economía Internacional y Fiscalidad. No contemplándose másteres y doctorados en campos tales como: Economía Social, Desarrollo Económico, Pensamiento Económico, etc.

Una vez presentadas las relaciones que se establecen entre educación y economía, y caracterizada el tipo de formación en las áreas de las Ciencias Económicas y Empresariales, en el siguiente apartado se profundiza en la formación en Economía Social, caracterizándola y destacando la importancia que puede tener para la promoción de este tipo de economía.

\subsection{Caracterización de la formación en Economía Social}

\subsubsection{Delimitación de la Economía Social}

En el presente apartado se conceptualiza a la economía social con objeto de diferenciarla de la economía de mercado capitalista y delimitar, de esta forma, nuestro objeto de estudio, sobre todo a la hora de identificar la oferta formativa que aborda esta rama de la Economía.

Para empezar, cabe señalar que son varios los organismos que han llevado a cabo la tarea de definir lo que se entiende por Economía Social (CNLAMCA, 1982; CWES, 1990). De todas estas definiciones, destaca la ofrecida por el Centro Internacional de Investigación e Información sobre la Economía Pública, Social y Cooperativa (CIRIEC) en 2006, según la cual:

“La Economía Social es el conjunto de empresas privadas organizadas formalmente, creadas para satisfacer las necesidades de sus socios a través del mercado, produciendo bienes y servicios, asegurando o financiando, y en las que la eventual distribución de beneficio excedente así como la toma de decisiones, no están ligadas directamente con el capital o con las cotizaciones aportados por cada socio, correspondiendo un voto a cada uno

\footnotetext{
${ }^{3}$ Para profundizar en el análisis de la estructura de los planes de estudio de Economía y Empresa puede consultarse a Peña y Caso (2006).
}

REVESCO No 121 - Segundo Cuatrimestre 2016 - ISSN: 1885-8031 - www.ucm.es/info/revesco 
de ellos. La Economía Social también agrupa a aquellas entidades privadas organizadas formalmente para con autonomía de decisión, y libertad de adhesión que producen servicios de no mercado a favor de las familias, cuyos excedentes, si los hubiera, no pueden ser apropiados por los agentes económicos que las crean, controlan o financian” .

De este modo, en esta definición se pueden distinguir dos grandes grupos dentro de la Economía Social (Barea, 1990): el subsector de mercado, compuesto por aquellos agentes de la Economía Social cuyos recursos provienen de las ventas realizadas en el mercado, y el subsector de no mercado, compuesto por aquellas entidades privadas cuya producción se distribuye de forma gratuita o a precios significativos, no distribuyen beneficios, y obtienen recursos de los hogares (donaciones o cuotas de socios), de pagos de las administraciones públicas y de rentas de la propiedad.

Cabe destacar que a la hora de afrontar el estudio de las entidades de Economía Social existen tres enfoques diferentes. El primero de ellos es el conocido como enfoque Nonprofit. Dicho enfoque tiene origen anglosajón e identifica a la Economía Social con las entidades que cumplen las siguientes cinco características (Salamon y Anheier, 1998): 1) son organizaciones formales, 2) privadas,3) que se autogobiernan, 4) que cuentan con voluntariado, y 5) que en ningún caso distribuyen el beneficio entre sus miembros, sino que lo destinan al cumplimiento de sus objetivos (constructo de no distribución). Es decir, son entidades sin ánimo de lucro con un fin social. Las entidades más representativas son las fundaciones y las charities anglosajonas. Este enfoque se identifica así con el subsector de no mercado al que se refería el CIRIEC (2006).

Por otro lado, el enfoque de la Economía Social tiene origen francés y se ha extendido a otros países de Europa, Latinoamérica y Canadá. Este enfoque considera dentro del estudio de la Economía Social a las entidades que, como se señaló en la definición del CIRIEC en el subsector de mercado (2006), cumplen los siguientes principios: 1) entidades privadas, 2) organizadas formalmente (con personalidad jurídica), 3) con autonomía de decisión, 4) con libertad de adhesión, 5) con eventual distribución de beneficios no vinculada al capital, 6) que ejercen una actividad económica en sí misma para satisfacer las necesidades de los miembros que la componen o de la sociedad en general, y 7) son democráticas. De este modo, existen diferencias entre los enfoques Nonprofit y el de Economía Social, entre las que destacamos la existencia de un principio democrático y la posibilidad de distribución de beneficios (siempre con límites) (Defourny y Develtere, 2009). El constructo de no distribución de beneficios que 
contempla el enfoque de las Nonprofit es considerado por los autores como demasiado estricto, ya que no incluiría en su campo de estudio a las cooperativas, calificadas como la columna vertebral de la Economía Social (Monzón, 1991). Por este motivo, el enfoque de la Economía Social es valorado como superior al Nonprofit, ya que es más amplio y engloba a más tipos de entidades (Defourny y Develtere, 2009).

Por último, en la última década ha aparecido un nuevo enfoque que va adquiriendo cada vez más fuerza: el de las empresas sociales y el emprendimiento social. Estas empresas se caracterizan por servir a un objetivo social independientemente de su forma jurídica (Nyssens, 2006). Es decir, se trata de empresas que proveen servicios que el Estado no suministra o lo hace de forma insuficiente (Defourny y Nyssens, 2012).

Una vez realizado el repaso sobre la concepción de la Economía Social y sus características, así como sobre los diferentes campos de estudio que engloba, nos encontramos en disposición de definir y caracterizar la formación universitaria española de posgrado en materia de Economía Social. De tal forma que consideraremos en nuestro análisis, dentro de esta formación, a todas aquellas titulaciones en las que, en su denominación, aparezcan algunos de los siguientes conceptos: Economía Social y/o Economía Solidaria, Organizaciones Sociales y/o Organizaciones No Gubernamentales, Tercer Sector, Sector No Lucrativo, Cooperativas, empresas sociales emprendimientos y/o innovaciones sociales.

\subsubsection{Características e importancia de la formación en el fomento de la Economía Social}

Tal y como recoge Fajardo (2012), el fomento de la economía social, a través de su integración en las políticas públicas, debería ser un objetivo de los Estados de la Unión Europea, según la Resolución del Parlamento Europeo sobre la Economía Social de $2009^{4}$.

Por otro lado, como se indica en el Informe de la Subcomisión para el Fomento de la Economía Social (Congreso de los Diputados, 2011), es necesaria la iniciativa de la política en la economía social, donde su propia pluralidad y diversidad hacen posible la construcción de un nuevo tipo de institucionalidad. De hecho, como recogen Chaves et al (2013: 136), la forma de cristalizar el reconocimiento institucional de una realidad socioeconómica es a través de su consideración en la normativa, recogiéndola en políticas públicas generales y

\footnotetext{
${ }^{4}$ España integra la dimensión de la Economía Social en sus políticas públicas desde la Orden de 21 de febrero de 1986, que estableció determinadas ayudas para la creación y mantenimiento de sociedades cooperativas y laborales, así como para la difusión y el desarrollo del cooperativismo y la economía social.
} 
específicas, estableciendo órganos públicos especializados dedicados a esta realidad económica, y sobre todo aprobando leyes que la regulan.

En este sentido, las medidas de fomento de la economía social se pueden clasificar a través de la definición de las siguientes tipologías de políticas públicas (Chaves, 2010: 6):

a) Políticas específicas, generales y excluyentes: las primeras van dirigidas exclusivamente a la economía social, las segundas a cualquier tipo de empresas y las terceras excluyen explícitamente o económicamente a empresas de economía social.

b) Políticas transversales, dirigidas a toda la economía social y políticas sectoriales, dirigidas a un tipo de empresa de economía social.

c) Según los instrumentos utilizados se pueden diferenciar entre políticas de oferta, que se dirigen a la estructura de las empresas de economía social, y políticas de demanda, cuyo objetivo es incidir en el volumen de actividad económica de las empresas de economía social, promoviendo su contratación.

d) Las políticas de oferta pueden, a su vez, articularse en dos niveles, como políticas institucionales, dirigidas a institucionalizar a la economía social, reconociendo su identidad diferenciada, y como políticas de difusión, formación e investigación.

Siguiendo esta clasificación, podría decirse que el fomento de la economía social a través de la educación y de la formación pública, objeto del presente estudio, se define como una política pública específica, transversal y de oferta, tanto institucional, ya que debe ser reconocida en toda la oferta pública educativa, como de formación.

Tabla 1. Clasificación de las políticas de formación en Economía Social

\begin{tabular}{|c|c|c|c|}
\hline Criterios & \multicolumn{3}{|c|}{ Tipos de políticas (denominación) } \\
\hline Fomento o no de la E.S. & Específicas & Generales & Excluyentes \\
\hline Ámbito fomento de la E.S. & Transversales & \multicolumn{2}{|c|}{ Sectoriales } \\
\hline Instrumentos fomento E.S. & Oferta & \multicolumn{2}{|c|}{ Demanda } \\
\hline Objetivo del fomento E.S. & Institucional & \multicolumn{2}{|c|}{ Difusión } \\
\hline
\end{tabular}

Fuente: elaboración propia a partir de Chaves (2010)

Sin embargo, respecto a las políticas públicas de formación, se puede destacar cómo, aún considerando la importancia que pueda tener la formación en Economía Social como política pública que contribuya a impulsar y dinamizar esta forma diferente de entender la 
economía, podemos decir que, tal y como se ha apuntado en el apartado anterior, está escasamente desarrollada. De hecho, en el panorama universitario español no existe una titulación de grado para los cuadros de mandos directivos de las organizaciones del tercer sector y/o de la economía social, encontrándose escasamente desarrollados los estudios en Economía Social (Moyano Fuentes, Bruque Cámara y Matínez Segovia, 2002; Gelambi, 2006; CEPES, 2012; Juliá (2013), Martínez López, et. al, 2013; entre otros).

En este sentido, tal y como recoge CEPES (2012), del análisis de la oferta formativa universitaria de posgrados en Economía Social se puede destacar: a) la existencia de escasos cursos de formación de posgrado y especialización sobre gestión y dirección de empresas de economía social; b) la mayor parte de estos cursos son de modalidad presencia o semipresencial; c) las propias organizaciones de empresas de economía social son promotoras principales de buena parte de esta oferta formativa; d) escasos cursos de doctorados y escasas líneas de investigación sobre la temática.

Por otra parte, Moyano Fuentes et. al (2002: 291) concluyen que, aunque la presencia de la economía social en las universidades españolas es medianamente satisfactoria, en los territorios con una cultura más cooperativa, tales como Cataluña o Andalucía, estos estudios aún están escasamente desarrollados. Además, se echa en falta que este tipo de enseñanza y asignaturas adquieran un carácter obligatorio ${ }^{5}$. Por otro lado, mientras que en los estudios de grados se imparten materias que versan sobre temas jurídicos, en los posgrados adquieren una mayor importancia los aspectos económicos.

Sin embargo, Moyano Fuentes et. al (2002) señalan como una de las limitaciones de su estudio, el no haber analizado los contenidos y programas de las diferentes asignaturas y cursos, con objeto de profundizar en los aspectos cualitativos de esas enseñanzas, lo cual intentamos abordar con este primer estudio de carácter exploratorio y descriptivo.

Por otro lado, a pesar de que en España existen varios centros y grupos de investigación específicos de Economía Social, agrupados la mayoría de ellos en la Red Enuies, Martínez López, et al (2013:93) afirman que las empresas de participación y de economía social son grandes desconocidas, y que la falta de información provoca que los jóvenes decidan crear empresas bajo otras formas jurídicas. Además, estas empresas, en

\footnotetext{
${ }^{5}$ Ello se relaciona, tal y como quedó recogido en el epígrafe anterior, con las características fundamentales de la formación universitaria en el área de las Ciencias Económicas, recogidas en los citados libro blancos de los estudios del Grado de Economía y del de ADE.
} 
épocas de crisis, adquieren especial importancia como generadoras de rentas, lo cual hace necesario la promoción y formación en este tipo de empresas desde los niveles educativos inferiores hasta la universidad.

Por tanto, a partir del análisis de la escasa bibliografía que existe al respecto, podemos concluir que, a pesar de la importancia que tiene la institucionalización de la economía social en general, y la implementación de políticas públicas de educación y formación en particular, aún son escasos los programas formativos que la contemplan, así como su institucionalización y reconocimiento político. No en vano, tal y como se ha apuntado, las asignaturas de carácter obligatorio son todavía escasas, no encontrándose titulaciones específicas de grados relacionadas con la economía social.

Para corroborar estas conclusiones teóricas y contrastar las diferencias que puedan existir entre programas formativos de posgrado orientados hacia estas formas diferentes de entender la economía, en el siguiente epígrafe se analiza la oferta formativa universitaria española de posgrados, relacionados con las áreas de las Ciencias Económicas y Empresariales, prestando especial atención a la formación en materia de Economía Social.

\section{OFERTA ESPAÑOLA DE POSGRADOS EN TEMAS ECONÓMICOS. ESPECIAL REFERENCIA A LA ECONOMÍA SOCIAL}

\subsection{Metodología del estudio empírico}

El presente estudio empírico tiene dos grandes objetivos: a) por un lado, analizar el tipo de formación de posgrado que se imparte en las universidades españolas en las áreas de las Ciencias Económicas y Empresariales; y b) por otro, analizar las características de la formación de posgrado en Economía Social para caracterizarla y diferenciarla del resto. Para ello, el trabajo empírico se ha dividido en dos grandes fases:

a) En una primera se han analizado todos los títulos de posgrado que, relacionados con las Ciencias Económicas y Empresariales (Máster Oficial, Máster Propio, Doctorado y Curso de Experto Universitario), se imparten en el Curso Académico 2014/2015 en las Universidades Españolas.

Esta consulta se ha realizado por Internet, a partir de la página web de la Conferencia de Rectores de las Universidades Españolas (CRUE, 2014). A través de estas consultas se ha elaborado una base de datos en Excel en la que quedaron registrados los siguientes 
campos: universidad consultada, comunidad autónoma a la que pertenecía, denominación de la titulación, tipo de titulación, contacto, página web del título y si está o no relacionado con la economía social.

Para integrar una titulación dentro de la formación en economía social se utilizó el criterio recogido en el epígrafe anterior, es decir, que en su denominación aparecieran algunos de los siguientes conceptos: a) Economía Social y/o Economía Solidaria; b) Organizaciones Sociales y/o Organizaciones No Gubernamentales; c) Tercer Sector; d) Empresas Sociales; y/o e) Emprendimientos y/o Innovaciones Sociales; y/o f) Cooperativas; y/o g) Sector No Lucrativo. El resto de titulaciones relacionadas con las Ciencias Económicas y Empresariales, e integradas en dicha base de datos, no tratan a la economía social o, si es así, lo hacen de una forma tangencial, no siendo el objetivo central de la titulación.

b) En una segunda parte del estudio se han analizado, a partir de un cuestionario estructurado, aplicando el paquete estadístico del SPSS, las características de la formación impartida en los diferentes títulos de posgrado identificados en la fase anterior, prestando especial atención a la formación en economía social y a sus diferencias respecto a los restantes títulos, más relacionados con la administración y dirección de empresas convencionales, así como con el emprendimiento e innovación en las mismas.

\subsection{Resultados del estudio empírico}

\subsubsection{Caracterización de la formación de posgrado en Ciencias Económicas y Empresariales}

Como resultado de la consulta realizada a través de la web de la CRUE, de todas las universidades españolas, públicas y privadas, se obtuvieron un total de 816 títulos de posgrados relacionados con la gestión, la economía, la administración y dirección de empresa y el desarrollo económico (desarrollo territorial, desarrollo sostenible, cooperación al desarrollo, etc.).

De todas estas titulaciones, siguiendo el criterio anteriormente apuntado, puede decirse que, tal y como se recoge en la Tabla 2, sólo alrededor del 4\% (3,92\%) están referidas directamente a la economía social y alrededor del 58\% (57,84\%) están relacionadas con la administración y dirección de empresas, así como con el emprendimiento y la innovación en las mismas. Por su parte, un $38,24 \%$ se corresponde con titulaciones relacionadas con la 
economía y el desarrollo económico en general, así como con la gestión de determinados tipos de empresas y sectores económicos (turismo, moda, transporte y logística, servicios sociales, etc.). De tal forma que, en este último grupo de titulaciones, se puede impartir, o no, de forma tangencial algún tipo de formación relacionada con la economía social. No obstante, puede afirmarse que el objetivo central de estas titulaciones no está relacionado con la economía social, motivo por el cual no se han incluido en la primera columna de la Tabla 2.

Tabla 2. Titulaciones de Posgrado en España relacionadas con las Ciencias Económicas y Empresariales

\begin{tabular}{|l|c|c|c|}
\hline & $\begin{array}{c}\text { Economía Social y } \\
\text { emprendimientos e } \\
\text { innovaciones sociales }\end{array}$ & $\begin{array}{c}\text { Administración y } \\
\text { Dirección de } \\
\text { Empresas, } \\
\text { emprendimientos e } \\
\text { innovación }\end{array}$ & $\begin{array}{c}\text { Economía, Desarrollo } \\
\text { y gestión de } \\
\text { determinados } \\
\text { sectores y empresas }\end{array}$ \\
\hline Número titulaciones & 32 & 472 & 312 \\
\hline Porcentajes & $3,92 \%$ & $57,84 \%$ & $38,24 \%$ \\
\hline
\end{tabular}

Fuente: elaboración propia

Por tanto, a raíz de este análisis confirmamos, tal y como apuntábamos en el análisis bibliográfico, que la presencia de la economía social en la formación universitaria económica de posgrado en España es testimonial, al representar tan sólo un $4 \%$ de las titulaciones relacionadas con estas ramas del conocimiento.

Sin embargo, cabe destacar, tal y como también se ha apuntado en el marco teórico, cómo son mayoría las titulaciones relacionadas con la administración y dirección de empresas, así como las de fomento del emprendimiento y la innovación en este tipo de entidades, llegando a representar casi el $60 \%$ de las titulaciones de posgrado. Ello se corresponde con el actual paradigma bajo el cual se organiza la economía actual, basada en el mercado capitalista, donde las empresas convencionales capitalistas y, por tanto, el emprendimiento e innovación en este tipo de empresas, dominan en la organización económica de la sociedad actual.

En este sentido, queda también justificado el hecho de que las titulaciones relacionadas con la economía y el desarrollo económico, en general, representen tan sólo el $38 \%$ de la oferta formativa. Este porcentaje se hace aún más reducido si tenemos en cuenta que en este tipo de oferta formativa se integran todas aquellas titulaciones relacionadas con la gestión de empresas pertenecientes a determinados sectores económicos, tales como: el 
turismo, el deporte, la moda, la logística y el transporte, la dependencia, etc.; ya que en la gran mayoría de éstas se siguen los criterios de gestión que impone el actual sistema económico.

Una vez cuantificada y puesta de relieve el tipo de formación de posgrado en Ciencias Económicas y Empresarailes que se imparte en las universidades españolas, en el siguiente apartado se analiza más en profundidad, a partir de una encuesta estructurada, las características, más de corte cualitativo, de este tipo de formación, haciendo especial hincapié en la economía social, realizando un análisis comparado respecto al resto de titulaciones.

\subsubsection{La Economía Social en la formación de posgrado española}

Para analizar qué presencia y de qué forma se enseña la economía social en los estudios de posgrado, se diseñó y validó un cuestionario relacionado con diferentes aspectos sobre dicha materia. Este cuestionario fue enviado a las 816 universidades incluidas en la base de datos que se construyó en la fase 1 de la investigación. De los 816 cuestionarios que enviamos vía email a todas las universidades incluidas en dicha base de datos, sólo 30 lo rellenaron; por lo que el índice de respuesta es de un 3,7\%. La Tabla 3 refleja las características de la muestra obtenida.

Este porcentaje de respuesta fue bajo, por lo que los resultados deben ser tomados con mucha precaución, si bien, tal y como se viene recogiendo en este trabajo, se trata de una investigación de corte exploratoria, que nos puede dar una primera aproximación a las diferentes metodologías que se aplican en la formación en economía social, respecto a las restantes titulaciones, sobre la cual será necesario profundizar

Como se puede observar, la mayoría de las respuestas obtenidas versan sobre másteres oficiales $(56,7 \%)$, seguidas de másteres propios $(26,7 \%)$, de programas de doctorado $(13,3 \%)$ y de títulos de experto (3,3\%). La media del número de ediciones de todos ellos es de 5,54. Por su parte, el $60 \%$ de los encuestados afirman poseer un enfoque profesional en sus estudios de posgrado, mientras que sólo un $10 \%$ afirman tener un enfoque investigador y un $30 \%$ ambos tipos de enfoque. Por otro lado, el $77 \%$ de los encuestados pertenecen a universidades públicas y el $23 \%$ a privadas.

Por último, según el título del estudio, el 16,7\% son estudios específicos de economía social y emprendimientos e innovaciones sociales (ES) (de los cuales el $80 \%$ pertenecen a universidades públicas y el $20 \%$ a universidades privadas), el $70 \%$ de emprendimiento, 
innovación y administración y dirección de Empresas, más en la línea del sistema económico dominante (EC), y el 13,3\% son de economía, desarrollo y gestión en determinados sectores y empresas (EyD), los cuales, como ya se comentó anteriormente, pueden tratar temas de economía social de forma tangencial, como ya se mencionó en líneas anteriores. A pesar de realizar esta diferenciación, cabe señalar que, a la hora de analizar las respuestas del cuestionario, nos hemos centrado en las diferencias de los programas de estudio que, por su título, pertenecían más a la corriente neoclásica (EC) y a la economía social (ES), pues es donde, a priori, parece que pueden existir unas diferencias más significativas a este respecto.

Tabla 3. Características de la muestra $(\mathrm{N}=30)(\%)$

\begin{tabular}{|c|c|c|}
\hline \multirow{4}{*}{ Tipo de título } & Experto & 3,3 \\
\cline { 2 - 3 } & Máster propio & 26,7 \\
\cline { 2 - 3 } & Máster oficial & 56,7 \\
\cline { 2 - 3 } & Doctorado & 13,3 \\
\hline \multirow{3}{*}{ Enfoque } & Número medio de ediciones & 5,54 \\
\cline { 2 - 3 } & profesional & 60 \\
\hline \multirow{2}{*}{$\begin{array}{c}\text { Tipo } \\
\text { Universidad }\end{array}$} & investigador & 10 \\
\hline \multirow{3}{*}{$\begin{array}{c}\text { Tipo de } \\
\text { titulación según } \\
\text { contenido }\end{array}$} & ambos & 30 \\
\cline { 2 - 3 } & Pública & 23 \\
\cline { 2 - 3 } & Economía, Desarrollo y gestión en & 13,3 \\
\cline { 2 - 3 } & Ecomía Social y emprendimientos e & 16,7 \\
\cline { 2 - 3 } & Emprendimiento, innoviones sociales (ES) & 70,0 \\
\hline
\end{tabular}

Fuente: elaboración propia

Para conocer las diferencias que pueden existir entre los títulos afines a la Economía Social (ES) y aquéllos más afines a la Economía de Mercado Capitalista (EC), en el cuestionario se incluyeron preguntas destinadas tanto a detectar la presencia de la economía social, como a conocer otras diferencias que pueden suponer puntos clave a la hora de que los futuros actores desempeñen su labor en el sistema económico vigente. De esta forma, se preguntó a los encuestados en primer lugar en qué medida estaban presentes en los programas de estudios los siguientes aspectos: a) fomentar el emprendimiento, b) técnicas de gestión empresarial, c) gestión de ONGs, d) analizar el papel de la Economía Social dentro de la 
Economía y del desarrollo Económico. La respuesta se ofrecía en forma de porcentaje respecto al $100 \%$ del plan de estudios. La Tabla 4 muestra los resultados obtenidos a este respecto, una vez calculadas las medias y realizada la prueba $\mathrm{T}$ para comprobar si existen diferencias significativas entre ambos grupos de estudio.

Tal y como se puede observar, en cuanto al fomento del emprendimiento las medias son muy dispares en ambos grupos, siendo bastante superior en las EC (18,8\% en los ES y $40,9 \%$ en los EC). Aunque dichas diferencias no son significativas, parece que en los estudios específicos de economía social no se promueve tanto el emprendimiento como en los estudios tradicionales, por lo que sería una debilidad de los ES a la hora de impulsarla y dinamizarla. No obstante, habría que profundizar en el estudio sobre dicha temática. En cuanto a las técnicas de gestión empresarial no hay diferencias importantes, pero sí en la gestión de las ONGs, pues los estudios de EC sólo dedican un 7,6\% de sus programas a enseñar este tipo de contenidos, frente a un 14,6\% en los de ES. De este modo, a pesar de que esta diferencia no sea significativa en nuestro estudio, parece que habría que tenerla en cuenta para futuras investigaciones. Por último, respecto al análisis del papel de la economía social dentro de la economía y del desarrollo económico, sí existen diferencias significativas entre las medias de los porcentajes que dedican los dos grupos considerados en sus programas de estudio: $24 \%$ en el caso de los ES y 4,4\% en el de los EC. Esta diferencia es significativa al 99\% de confianza, lo cual no es sorprendente si tenemos en cuenta que el grupo de la ES está expresamente dedicado a la economía social.

Tabla 4. Prueba T sobre aspectos incluidos en os títulos de ES y EC

\begin{tabular}{|c|c|c|c|c|c|c|c|}
\hline $\begin{array}{l}\% \text { programa que } \\
\text { dedica a los } \\
\text { siguientes aspectos: }\end{array}$ & ES/EC & $\mathbf{N}$ & Media & Des. típ. & $\begin{array}{l}\text { Error típ. } \\
\text { de la } \\
\text { media }\end{array}$ & $\mathbf{t}$ & $\begin{array}{l}\text { Sig. } \\
\text { (bilatera } \\
\text { l) }\end{array}$ \\
\hline \multirow{2}{*}{$\begin{array}{l}\text { Fomentar el } \\
\text { emprendimiento }\end{array}$} & ES & 5 & 18,8 & 8,07 & 3,61 & \multirow{2}{*}{,- 41} & \multirow{2}{*}{,682 } \\
\hline & $\mathrm{EC}$ & 21 & 40,9 & 117,05 & 25,54 & & \\
\hline \multirow{2}{*}{$\begin{array}{l}\text { Técnicas de gestión } \\
\text { empresarial }\end{array}$} & ES & 5 & 30,2 & 25,49 & 11,40 & \multirow{2}{*}{,- 37} & \multirow{2}{*}{,718 } \\
\hline & EC & 21 & 34,5 & 23,37 & 5,10 & & \\
\hline \multirow{2}{*}{ Gestión de ONGs } & ES & 5 & 14,6 & 11,97 & 5,35 & \multirow{2}{*}{85} & \multirow{2}{*}{,403 } \\
\hline & EC & 21 & 7,6 & 17,36 & 3,79 & & \\
\hline Analizar el papel de & ES & 5 & 24,0 & 20,73 & 9,27 & 3,83 & 001 \\
\hline
\end{tabular}




\begin{tabular}{|c|c|c|c|c|c|c|c|}
\hline $\begin{array}{c}\text { la Economía Social } \\
\text { dentro de la } \\
\begin{array}{c}\text { Economía y el } \\
\text { desarrollo económico }\end{array}\end{array}$ & EC & 21 & 4,4 & 6,33 & 1,38 & & \\
\hline
\end{tabular}

Fuente: elaboración propia

A continuación, se pidió a los encuestados que valorasen en una escala de Likert de 1 a 7 en qué medida estaban considerados en sus programas de estudios los comportamientos emprendedores incluidos en la Tabla 5, siendo 1 "ninguna presencia" y 7 "máxima presencia". De todos ellos, la diferencia de medias sólo es significativa en la cooperación con un $95 \%$ de confianza, siendo la media superior en los estudios de ES (6,8 vs. 5,10). Por lo tanto, los títulos de economía social transmitirían más el comportamiento de cooperación que los títulos insertos en la corriente dominante. No en vano, estamos ante uno de los principios que caracteriza a la economía social.

Por otro lado, respecto a la orientación emprendedora (la cual incluye a la innovación, la proactividad y la asunción de riesgos) es siempre menor en las titulaciones de ES (5,0 vs. 5,$81 ; 5,4$ vs. 5,$95 ; 4,4$ vs. 4,67). Asimismo, el trabajo en equipo también es inferior en la ES $(5,8$ vs. 6,19$)$. Todo ello situaría a las titulaciones de ES en desventaja frente a las EC, ya que estos comportamientos implican el dinamismo de los negocios y la consecución de objetivos, del tipo que sea. No obstante, estas diferencias no son significativas, por lo que habría que seguir estudiando más en profundidad dichas variables. No en vano, como hipótesis de partida para posteriores estudios, encontraríamos en el escaso desarrollo de estas variables microeconómicas algunos de los inconvenientes que pueden impedir el desarrollo de estas entidades.

Por último, la detección de oportunidades de negocio y la detección de oportunidades de negocio derivadas de necesidades sociales es superior en las titulaciones de ES (4,8 vs. 4,38 y 4,40 vs. 2,95). Esta situación colocaría a las ES en una situación de ventaja frente a las EC, aunque, al igual que en el caso anterior, dichas diferencias tampoco son significativas.

Tabla 5. Prueba T sobre comportamientos emprendedores en ES y EC

\begin{tabular}{|c|c|c|c|c|c|c|c|}
\hline \multirow{2}{*}{ Comportamientos } & ES/EC & $\mathbf{N}$ & Media & $\begin{array}{c}\text { Des. } \\
\text { típ. }\end{array}$ & $\begin{array}{c}\text { Error } \\
\text { típ. de } \\
\mathbf{l a} \\
\text { media }\end{array}$ & $\mathbf{t}$ & $\begin{array}{c}\text { Sig. } \\
\text { (bilateral) }\end{array}$ \\
\hline \multirow{2}{*}{ Innovación } & ES & 5 & 5,00 & 2,000 &, 894 & \multirow{2}{*}{,- 972} & \multirow{2}{*}{, 341} \\
\cline { 2 - 7 } & EC & 21 & 5,81 & 1,601 &, 349 & &, 467 \\
\hline Proactividad & ES & 5 & 5,40 & 1,140 &, 510 &,- 739 & \multirow{2}{*}{} \\
\hline
\end{tabular}




\begin{tabular}{|c|c|c|c|c|c|c|c|}
\hline & $\mathrm{EC}$ & 21 & 5,95 & 1,564 & ,341 & & \\
\hline \multirow{2}{*}{ Asunción de riesgos } & ES & 5 & 4,20 & 2,168 & ,970 & \multirow{2}{*}{,- 515} & \multirow{2}{*}{,611 } \\
\hline & EC & 21 & 4,67 & 1,742 & ,380 & & \\
\hline \multirow{2}{*}{ Cooperación } & ES & 5 & 6,80 &, 447 & ,200 & \multirow{2}{*}{2,192} & \multirow{2}{*}{, 038} \\
\hline & EC & 21 & 5,10 & 1,700 & ,371 & & \\
\hline \multirow{2}{*}{ Trabajo en equipo } & ES & 5 & 5,80 & 2,168 & ,970 & \multirow{2}{*}{,- 523} & \multirow{2}{*}{,606 } \\
\hline & EC & 21 & 6,19 & 1,327 & 290 & & \\
\hline \multirow{2}{*}{$\begin{array}{c}\text { Detección de oportunidades } \\
\text { de negocio }\end{array}$} & ES & 5 & 4,80 & 1,483 & ,663 & \multirow{2}{*}{,401 } & \multirow{2}{*}{,692 } \\
\hline & EC & 21 & 4,38 & 2,202 & ,480 & & \\
\hline \multirow{2}{*}{$\begin{array}{c}\text { Detección de oportunidades } \\
\text { de negocio derivadas de } \\
\text { necesidades sociales }\end{array}$} & ES & 5 & 4,40 & 2,408 & 1,077 & \multirow{2}{*}{1,325} & \multirow{2}{*}{, 198 } \\
\hline & EC & 20 & 2,95 & 2,139 & ,478 & & \\
\hline
\end{tabular}

Fuente: elaboración propia

La siguiente cuestión en analizar es la concepción de la economía social en el sistema económico vigente, cuyos resultados se muestran en la Tabla 6. Como se puede observar, un $46 \%$ de los estudios de EC perciben la economía social como complementaria a la Economía de Mercado Capitalista, y un 26\% como complementaria al Estado. Por su parte, un $25 \%$ de los títulos de ES advierten a la Economía Social como complementaria a la Economía de Mercado Capitalista y otro 25\% como complementaria al Estado. Asimismo, un $50 \%$ de los estudios ES reconocen a la Economía Social como sustitutiva/alternativa a la Economía de Mercado Capitalista, frente a un 20\% de las EC. De esta manera, los resultados apuntan a que las titulaciones ES presentan una concepción de la Economía Social más sustancial y radical que las titulaciones EC, donde la Economía Social es percibida como una alternativa al sistema económico dominante.

Tabla 6. Concepción de la Economía Social en el sistema económico vigente

\begin{tabular}{|l|l|l|}
\hline Función de la Economía Social en el Sistema Económico & ES & EC \\
\hline Complementaria a la Economía Capitalista & $25,0 \%$ & $46,7 \%$ \\
\hline Complementaria al Estado & $25,0 \%$ & $26,7 \%$ \\
\hline Sustitutiva/alternativa a la Economía Capitalista & $50,0 \%$ & $20 \%$ \\
\hline Perdidos & $0,0 \%$ & $6,7 \%$ \\
\hline Total & $\mathbf{1 0 0 , 0 \%}$ & $\mathbf{1 0 0 , 0 \%}$ \\
\hline
\end{tabular}

Fuente: elaboración propia

Por último, se pidió a los encuestados que puntuaran en una escala de Likert de 1 a 7 en qué medida estaban presentes los valores propios de la Economía Social en sus planes de estudios, siendo 1 "ninguna presencia" y 7 "máxima presencia". Las medias de los resultados 
obtenidos para cada valor se encuentran disponibles en la Tabla 7, así como la prueba T que analizaba la significatividad de las diferencias entre las mismas.

Como se puede observar, en todos los valores considerados, las titulaciones en ES presentan una media superior a las de EC. De este modo, si nos detenemos en las medias, las titulaciones de ES conceden más importancia a la democracia, al trabajo en equipo, a la formación y la especialización, a la equidad, a la autofinanciación y al voluntariado que las EC (6,4 vs. 3,$86 ; 6,4$ vs. 6,$05 ; 4,8$ vs. 4,$57 ; 5,6$ vs. 3,$62 ; 5$ vs. 4,$14 ; 4,0$ vs. 2,95 respectivamente). Sin embargo, sólo presentan una diferencia significativa (al 95\% de confianza): la democracia participativa y la equidad $(6,4$ vs. 3,86 y 1,949 vs. 1,857$)$. Por lo tanto, estos resultados parecen respaldar el hecho de que los estudios específicos en economía social intentan transmitir los valores en los que ésta se basa. No obstante, tal y como hemos apuntado anteriormente para otras variables, habría que seguir profundizando en esta temática y repetir el estudio con una muestra mayor para conocer si sólo en estos dos valores se producen dichas diferencias significativas o también en el resto.

Tabla 7. Prueba T sobre valores propios de la Economía Social en ES y EC

\begin{tabular}{|c|c|c|c|c|c|c|c|}
\hline $\begin{array}{l}\text { Valores propios de la } \\
\text { Economía Social }\end{array}$ & ES/EC & $\mathbf{N}$ & Media & $\begin{array}{c}\text { Desviación } \\
\text { típ. }\end{array}$ & $\begin{array}{c}\text { Error } \\
\text { típ. de } \\
\text { la } \\
\text { media }\end{array}$ & $\mathbf{t}$ & $\begin{array}{c}\text { Sig. } \\
\text { (bilateral) }\end{array}$ \\
\hline \multirow{2}{*}{$\begin{array}{l}\text { Democracia } \\
\text { participativa }\end{array}$} & $\mathrm{ES}$ & 5 & 6,40 & ,894 &, 400 & \multirow{2}{*}{2,434} & \multirow{2}{*}{,023 } \\
\hline & $\mathrm{EC}$ & 21 & 3,86 & 2,265 &, 494 & & \\
\hline \multirow[t]{2}{*}{ Trabajo en equipo } & $\mathrm{ES}$ & 5 & 6,40 & ,894 & , 400 & \multirow{2}{*}{0,577} & \multirow{2}{*}{,569 } \\
\hline & $\mathrm{EC}$ & 21 & 6,05 & 1,284 & ,280 & & \\
\hline \multirow{2}{*}{$\begin{array}{l}\text { Formación y } \\
\text { profesionalización }\end{array}$} & ES & 5 & 4,80 & 1,924 & ,860 & \multirow{2}{*}{0,219} & \multirow{2}{*}{,829 } \\
\hline & $\mathrm{EC}$ & 21 & 4,57 & 2,135 & ,466 & & \\
\hline \multirow[t]{2}{*}{ Equidad } & ES & 5 & 5,60 & 1,949 & ,872 & \multirow{2}{*}{2,126} & \multirow{2}{*}{, 044} \\
\hline & $\mathrm{EC}$ & 21 & 3,62 & 1,857 & ,405 & & \\
\hline \multirow[t]{2}{*}{ Autofinanciación } & ES & 5 & 5,00 & 2,345 & 1,049 & \multirow{2}{*}{0,817} & \multirow{2}{*}{,422 } \\
\hline & $\mathrm{EC}$ & 21 & 4,14 & 2,056 & ,449 & & \\
\hline \multirow[t]{2}{*}{ Voluntariado } & ES & 5 & 4,00 & 2,449 & 1,095 & \multirow[b]{2}{*}{0,938} & \multirow[b]{2}{*}{,358 } \\
\hline & $\mathrm{EC}$ & 21 & 2,95 & 2,202 &, 480 & & \\
\hline
\end{tabular}

Fuente: elaboración propia

En definitiva, a partir de este prediagnóstico de la formación de posgrado en economía social en España, se puede concluir que ésta es aún escasa y que, en los casos en los que se da, existen algunas diferencias cualitativas (unas más significativas que otras) respecto a otras titulaciones más directamente relacionadas con la Economía de Mercado Capitalista, tales 
como las dirección y administración de empresas convencionales y el emprendimiento y la innovación en éstas.

Ello pone de relieve, por tanto, cómo la formación en Economía no tiene por qué ser igual siempre y en todo momento. De tal forma, que las características de la misma (objetivos, valores, competencias, habilidades, etc.) va a depender del enfoque y la forma de entender y analizar esta ciencia social.

\section{CONCLUSIONES}

Este estudio tenía como objetivos, en primer lugar, reflexionar sobre la formación en Economía y, posteriormente, analizar el grado y tipo de presencia de la enseñanza de la economía social en los estudios económicos de posgrado en las universidades españolas. Para este último objetivo se ha elaborado una base de datos que contiene todas las titulaciones de posgrados relacionados con las Ciencias Económicas y Empresariales. Asimismo, se ha diseñado un cuestionario estructurado con el objetivo de conocer, desde un punto de vista más cualitativo, la caracterización de esa formación en economía social. Tras el análisis de los resultados se llega a las siguientes conclusiones:

- La formación de posgrado específica en economía social sólo representa alrededor del $4 \%$ de la oferta total de titulaciones del sistema universitario español en la rama de Económicas y Empresariales.

- Existen determinadas diferencias significativas en el tipo de formación entre este grupo de titulaciones y las que están más orientadas a formar en la corriente económica imperante (sistema de mercado capitalista). Estas características hacen referencia a aspectos tales como: los valores, la forma de entender el comportamiento de la economía y la importancia que debe tener la Economía Social dentro de ésta, las competencias y habilidades a adquirir por parte de los gestores, etc.

- Todo ello pone de relieve, por tanto, cómo la formación en Economía no tiene por qué ser igual siempre y en todo momento, de tal forma que las características de esa formación (objetivos, metodologías, valores, competencias, habilidades, etc.) va a depender del enfoque y la forma de entender y analizar la Economía como ciencia social. A continuación se recogen algunas conclusiones en lo que a estas diferencias respecta. 
- Las titulaciones convencionales, que están más en la línea con una economía de mercado capitalista, dedican un porcentaje de sus planes de estudio mínimo, o incluso residual, a explicar la economía social y su papel en la economía y el desarrollo económico, en general.

- Las titulaciones específicas en Economía Social, respecto al resto de titulaciones, fomentan menos el emprendimiento entre sus alumnos, por lo que sería conveniente afrontar esta debilidad y tomar medidas al respecto, tal y como apuntan algunos de los trabajos consultados en el marco teórico de la presente investigación.

- Las titulaciones específicas en Economía Social promueven más la cooperación que las propias de la economía de mercado capitalista. Esto las sitúa en una situación de ventaja respecto al resto de titulaciones convencionales, puesto que los actores que se están formando pondrán poner en práctica este comportamiento emprendedor junto con todos los beneficios que éste reporta.

- Existen indicios para pensar que la innovación, la proactividad, la asunción de riesgos y el trabajo en equipo están más presentes en los planes de estudio de las titulaciones más centradas en la mentalidad de mercado capitalista, lo cual se configura como inconveniente a la hora de impulsar y dinamizar a la economía social.

- Las titulaciones específicas en Economía Social presentan una conceptualización de la Economía Social más radical que las convencionales, percibiéndola más como sustituta de las empresas convencionales o capitalistas que como complementarias de las mismas.

- También existen indicios para considerar que valores propios de la economía social, tales como el trabajo en equipo, la formación, la autofinanciación y el voluntariado, están más presentes en este tipo de titulaciones, aunque los resultados no son concluyentes.

Por tanto, y como limitación del presente estudio, dado el reducido volumen de respuestas que hemos obtenido en esta primera fase exploratoria del mismo, podemos decir que estamos ante un prediagnóstico sobre la caracterización de la formación universitaria de posgrado en materia de Economía Social en España; si bien, parece que los resultados, que necesariamente deben ser contrastados y ampliados en posteriores investigaciones, dejan 
entrever la necesidad de llevar a cabo determinadas actuaciones para mejorar este tipo de formación. De tal forma, que la formación en materia de Economía Social se convierta en una estrategia más que contribuya a impulsar y dinamizar esta otra forma de entender la Economía. Entre estas actuaciones cabría destacar:

- Desde un punto de vista cuantitativo, la necesidad de impulsar, en mayor medida, los estudios en Economía Social, dado el reducido peso que tienen actualmente dentro del mapa español de titulaciones universitarias de posgrados en las áreas de las Ciencias Económicas y Empresariales.

- Desde un punto de vista cualitativo, la necesidad de que los contenidos de estos estudios profundicen, en mayor medida, en la dimensión microeconómica de la economía social, es decir, en los aspectos más relacionados con la gestión de este tipo de entidades, entre los que caben citar: el fomento del emprendimiento, la innovación, la proactividad, la asunción de riesgos, etc., prestando especial atención a los aspectos sociales que deben impregnar estas dimensiones microeconómicas.

En este sentido, no se trata de que los aspectos macroeconómicos de la economía social (importancia que tiene para el desarrollo, peso que tiene en la economía, diferencias respecto a la economía capitalista y otros tipos de economías, etc.) no estén presentes en la estructura curricular de estos estudios, sino de la necesidad de que los aspectos microeconómicos adquieran un mayor peso y protagonismo con el objetivo de formar a verdaderos gestores, expertos en este tipo de entidades, que contribuyan a esta forma diferente de "hacer" economía.

En definitiva, a partir de este estudio se ha puesto de manifiesto cómo la institucionalización de la formación pública universitaria en materia de economía social se hace realmente necesaria, dado el reducido peso que aún tiene y las diferencias cualitativas que se apuntan en lo que respecta a los contenidos formativos respecto a las titulaciones convencionales en materia económica. Máxime si se tiene en cuenta la importancia que adquiere la economía social, puesta de manifiesto en la revisión bibliográfica, para el progreso socieconómico y, sobre todo, en momentos, como los actuales, de profunda crisis económica. 


\section{BIBLIOGRAFÍA}

ALONSO RODRIGO, E. (2001) Fiscalitat de cooperatives i societats laboral. Barcelona: Generalitat de Catalunya, Instituto para la promoción y la formación de cooperativas.

ANECA (2005) Libro Blanco del Título de Grado en Economía y en Empresa. Madrid: Agencia Nacional de Evaluación de la Calidad y Acreditación.

BAREA, J. (1990) Concepto y agentes de la economía social. CIRIEC-España, Revista de Economía Pública, Social y Cooperativa, № 8, pp. 109-117.

CARRERAS, L. (2008) El sector de la Economía Social o Tercer Sector. Cataluña: Universidad Rovira i Virigili, disponible http://www.tdx.cat/bitstream/handle/10803/8759/capitulo1.pdf?sequence=2.

CEPES (2012) La Economía Social en los centros de estudios de posgrado. Madrid: Confederación de Entidades para la Economía Social.

CHAVES, R. et al (2013) La economía social en clave internacional. Cuantificación, reconocimiento institucional y visibilidad social en Europa, Iberoamérica y norte de África. REVESCO. Revista de Estudios Cooperativos, No 112, pp. 122-150. DOI: 10.5209/rev_REVE.2013.v112.43069.

CHAVES, R. (dir.) (2010) La economía social en las políticas públicas en España. Valencia: CIRIEC-España.

CHAVES, R. y SAVALL, T. (2013) La insuficiencia de las actuaciones políticas de fomento de cooperativas y sociedades laborales frente a la crisis en España. REVESCO. Revista de Estudios Cooperativos, No 113, pp. 61-91. DOI: 10.5209/rev_REVE.2014.v113.43383.

CIRIEC (2006) Estudio sobre la economía social en los países de la Unión Europea. Madrid: Centro Internacional de Investigación e Información sobre la Economía Pública, Social y Cooperativa (CIRIEC).

CNLAMCA (1982) La lettre de l' conomie sociale, Paris.

CONGRESO DE LOS DIPUTADOS (2011) Informe de la Subcomisión para el Fomento de la Economía Social. Madrid: Boletín Oficial de las Cortes, № 581.

CORAGgiO, J. L. (2011) Principios, Instituciones y Prácticas de la Economía Social y Solidaria, en ACOSTA, A. y MARTÍNEZ, E. (ed.): Economía social y solidaria. El trabajo antes que el capital. Quito: Abya Yala.

CUADRADO ROURA, J.R. (1995) Introducción a la Política Económica. Madrid: Mc Graw Hill. 
CRUE (2014) Conferencia de Rectores de Universidades Españolas, http://www.crue.org/Paginas/Inicio.aspx?Mobile=0 (diciembre de 2014).

CWES (1990) Rapport à l'Ex cutif $R$ gional Wallon sur le secteur del' conomie sociale, Namur: Conseil Wallon de l'Economie Sociale.

DEFOURNY, J. y DEVELTERE, P. (2009) The Social Economy: The Worldwide Making of a Third Sector, en DEFOURNY, J., DEVELTERE, P., FONTENEAU, B. y Nyssens, M. (eds.): The Worldwide Making of the Social Economy: Innovations and ChangesLeuven: ACCO.

DEFOURNY, J. y NYSSENS, M. (2012) El enfoque EMES de la empresa social desde una perspectiva comparada, CIRIEC, Revista de Economía Social, Pública y Cooperativa, № 75, pp. 7-34.

DELGADO MUNEVAR, W.G. (2012) Formación de los profesionales: dilema entre ética y economía, Finanzas y Política Económica, Vol. 4, № 2, pp. 15-24.

FAJARDO GARCÍA, G. (2012) El fomento de la economía social en la legislación española, REVESCO. Revista de Estudios Cooperativos, No 107, pp. 58-97. DOI: 10.5209/rev_REVE.2012.v107.38747.

GELAMBI, M. (2006) La formación de líderes y directivos de la Economía Social y/o Tercer Sector en España. Madrid: Centro de Investigación de Economía y Sociedad,

JEANTET, T. (2001) La economía social europea o la tentación de la democracia en todas las cosas. Valencia: CIRIEC-España.

JULIÁ, F.J. (2013) Tribuna de opinión: emprendimiento y universidad. Una referencia al caso de España y de la UPV, REVESCO. Revista de Estudios Cooperativos, No 113, pp. 7-27. DOI: http://dx.doi.org/10.5209/rev_REVE.2014.v113.43394.

KRUEGER (1991) Report of Comisión on Graduate Education in Economics. Journal of Economic Literature, XXIX, № 1035-1053.

LAVILLE, J. L et al. (Dir.) (1994) L'économie solidaire, une perspective internationale. París: Desclée de Brouwer.

LAVILLE, J. L. y GARCÍA JANÉ, J. (2009) Crisis Capitalistas y economía solidaria. Una economía que emerge como alternativa real. Barcelona: Icaria.

LEONTIEF, W. (1982) Academic Economic, Science, No 217, pp.106-117.

LÓPEZ, H.F. (2009) ¿Formación o adiestramiento?: a propósito del programa de economía de la UIS, Apuntes del CENES, Vol. 28, Nº 47, pp. 167-184.

MARTÍNEZ RODRÍGUEZ, F. (2013) Educación, neoliberalismo y justicia social. Madrid, Pirámide. 
MARTÍNEZ LÓPEZ, S., FERNÁNDEZ GUADAÑO, J., BEL DURÁN, P. y LEJARRIAGA PÉREZ DE LAS VACAS, G. (2013) Necesidad de medidas para impulsar la creación de las empresas de participación desde los diferentes niveles de la enseñanza, CIRIECEspaña, Revista de Economía Pública, Social y Cooperativa, № 78, pp. 71-99.

MISAS ARANGO, G. (2004) El campo de la economía y la formación de los economistas, Cuadernos de Economía, Vol. 23, № 40, pp.205-229.

MOYANO FUENES, J., BRUQUE CÁMARA, S. y MARTÍNEZ SEGOVIA, F. J. (2002) La enseñanza universitaria en empresas de participación. Estado de la cuestión, en VARGAS SÁNCHE, A. y LEJARRIAGA PÉREZ DE LAS VACAS, G. Las empresas de participación en Europa: el reto del S. XXI, Ed. Escuela de Estudios Cooperativos, Madrid, pp. 275-294.

MONTEIRO, H. y FERREIRA, A. (2006) A Comparation of the Undergraduate Economics Major in Europe and in the United States, MPRA, Paper $N^{\circ} 771$, noviembre.

NYSSENS, M. (ed.) (2006) Social Enterprise - At the Crossroads of Market, Public Policies and Civil Society. Londres y Nueva York: Routledge.

OCDE (2013) Policy Brief on Social Entrepreneuship. Entrepreneurial Activities in Europe. Luxembourg: Publications Office of the European Union.

PEÑA FERNÁNDEZ, C. y CASO PARDO, C. (2006) Los planes de estudio de Economía y ADE en España antes del Espacio Europeo de Educación Superior. Revista Asturiana de Economía, № 36, pp.33-73.

POLANYI, K. (1944) The Great Transformation. Boston: Beacon Press.

RAZETO, L. (1984) Las Donaciones y la Economía de Solidaridad. Santiago: PET.

SALAMON, L. M. y ANHEIER, H. K. (1998) Social Origins of Civil Society: Explaining the Nonprofit Sector Cross-Nationally, Voluntas. International Journal of Voluntary and Nonprofit Organizations, Vol. 9, № 3, pp. $213-248$. 